\title{
Fluorine F 18 ODS2004436
}

National Cancer Institute

\section{Source}

National Cancer Institute. Fluorine F18 ODS2004436. NCI Thesaurus. Code C131497.

A radioconjug ate containing the epidermal growth factor receptor (EGFR) kinase tracer ODS2004436 labeled with fluorine F 18 for potential tumor imaging using positron emission tomography (PET). Upon administration of fluorine F 18 ODS2004436, the ODS2004436 moiety selectively targets and binds to forms of EGFR that contain activating mutations. This allows the visualization of tumor cells expressing activating EGFR mutations using PET. This agent can also be utilized in the prediction of responsiveness to EGFR tyrosine kinase inhibitors (TKIs) and the selection of patients that respond well to those kinase inhibitors that specifically target forms of EGFR with activating mutations. EGFR-activating mutations play a key role in the development of certain tumors and in tumor resistance to a variety of antineoplastic agents. 